\title{
UM MODELO CONCEITUAL DA DECISÃO DE CRIAÇÃO DE ESCRITÓRIO DE PROJETOS NA ÁREA DE TI
}

\section{A CONCEPTUAL DECISION-MAKING MODEL FOR IT PROJECT MANAGEMENT OFFICES}

\section{ANDREA GIOVANII SPELTA}

Doutor pela Escola de Administração de Empresas de São Paulo da

Fundação Getulio Vargas (FGV).

Professor da Faculdade de Sistemas de Informação da Universidade Municipal de São Caetano do Sul (USCS). Avenida Goiás, 3.400, Barcelona - São Caetano do Sul - SP - Brasil - CEP 09550-051

E-mail: agspelta@hotmail.com

\section{ALBERTO LUIZ ALBERTIN}

Doutor pela Faculdade de Economia, Administração e Contabilidade da Universidade de São Paulo (USP). Professor titular da Escola de Administração de Empresas de São Paulo da Fundação Getulio Vargas (FGV). Avenida Nove de Julho, 2.029, $3^{\circ}$ andar, Bela Vista - São Paulo - SP - Brasil - CEP 01313-902 E-mail: albertin@fgr.br 


\section{RESUMO}

Escritórios de projetos (EP) são entidades organizacionais às quais são atribuídas diversas responsabilidades relacionadas ao gerenciamento de projetos da organização. Em virtude da aceleração dos investimentos em tecnologia de informação (TI) e do aumento da importância estratégica desses investimentos, a criação dessas entidades começou a se intensificar na década de I990. Muito embora grandes empresas tenham criado um EP na área de TI (EP-TI), outras optaram por não fazer isso. Este artigo apresenta um modelo conceitual dos direcionadores da decisão de criação de EP-TI, sugerindo que essa decisão depende da satisfação com a entrega dos projetos de TI, da satisfação com o controle do portfólio de projetos de TI, da importância estratégica do portfólio de projetos de TI e da opinião da direção da empresa sobre EP. O modelo foi desenvolvido indutivamente com base na literatura e em quatro estudos de casos. Esta pesquisa contribui para o desenvolvimento da teoria contextual de gerenciamento de projetos por meio do aumento da compreensão das situações em que a criação de EP-TI faz sentido e das situações em que não faz. Para a prática, o modelo conceitual pode ser uma ferramenta muito útil para executivos que estão considerando se devem ou não criar um EP-TI em suas empresas, ajudando-os a tomar decisões mais eficazes.

\section{PALAVRAS-CHAVE}

Escritório de projetos; PMO; Gerenciamento de projetos; Administração de sistemas de informação; Gerenciamento de projetos de tecnologia de informação.

\section{ABSTRACT}

Projects management offices (PMO) are organizational entities charged with various responsibilities concerned with the management of the organization's projects. Because of accelerated investment in information technology (IT) and 
the increased strategic importance of those investments, creation of such entities became more intense in the I990 outside the context of project-oriented firms. Although some large companies did create PMOs in their IT areas (IT-PMO), others chose not to. This article provides a conceptual model of the drivers of the decision to create an IT-PMO, suggesting that the decision depends on satisfaction with IT project deliveries, on the strategic importance of the IT projects portfolio, and on the management's opinion on POs. The model was inductively developed from incentives to create or not create PMOs as reported in the literature and found in four case studies with large privately-owned, non-projects oriented Brazilian companies. This study contributes to the development of contextual project management by improving the understanding of situations where creating an IT-PMO makes and does not make sense. In practice, the conceptual model may be a very helpful tool for executives considering whether or not to create an IT-PMO at their companies, assisting them in making more effective decisions.

\section{KEYWORDS}

Project management office; PMO; Project management; Management of information technology; Management of information technology projects.

\section{INTRODUÇÃO}

O crescimento constante que se observa nos investimentos em tecnologia de informação nas empresas (MEIRELLES, 2004) fez crescer a importância de gerenciar adequadamente os projetos gerados por esses investimentos. Em consequência, muitas empresas passaram a reconhecer a relevância para a competitividade de suas competências em planejar, executar e controlar o portfólio de projetos (JUGDEV, 2004; SRIVANNABOON, 2004; KERZNER, 2002) e começaram a adotar medidas para aprimorar essas competências. Uma das medidas frequentemente adotada é a criação de escritórios de projetos (EP), que são entidades organizacionais às quais são "[...] atribuídas diversas responsabilidades relacionadas ao gerenciamento centralizado e coordenado dos projetos sob seu domínio" (PROJECT MANAGEMENT INSTITUTE, 2004, p. 369, tradução nossa).

O conceito de ter um grupo de pessoas dedicado à gestão de projetos começou a emergir em organizações fora dos setores de engenharia e construção, no final dos anos i980 e início da década de I990 (ENGLUND; GRAHAN; DINS- 
MORE, 2003). O movimento em direção ao estabelecimento de EP ganhou força em I994, e as empresas de tecnologia de informação (TI) ou as áreas de TI das empresas foram proeminentes nesse movimento (DAI; WELLS, 2004). Entretanto, como veremos nos estudos de caso, ainda hoje se observam empresas de grande porte, com elevados investimentos em TI, que optaram por não criar escritórios de projetos em suas áreas de TI e decidiram seguir outros caminhos para aprimorar sua competência em gerenciamento dos projetos de TI.

A pesquisa acadêmica sobre EP é relativamente recente e ainda não abordou de forma aprofundada os direcionadores da decisão de criação de EP, de modo a explicar por que algumas grandes empresas decidem criar um EP, enquanto outras preferem não fazê-lo. Tendo em vista a importância do gerenciamento de projetos de TI e considerando "as vantagens de desenvolver teorias com escopo restrito" (SAUER; REICH, 2007, p. 2, tradução nossa), propomos a seguinte questão de pesquisa: "Quais são os direcionadores da decisão de criação de escritórios de projetos nas áreas de TI (EP-TI) em grandes empresas não orientadas a projetos?".

Neste artigo, apresentamos um modelo conceitual dos direcionadores dessa decisão. Com base na literatura e em quatro estudos de caso, propomos um modelo com quatro direcionadores: I. satisfação com as entregas dos projetos de TI, 2. satisfação com o controle do portfólio dos projetos de TI, 3. importância estratégica do portfólio de projetos de TI e 4. opinião da empresa sobre EP em geral.

Os resultados desta pesquisa são relevantes tanto para a academia como para a prática. Para a academia, o modelo contribui para o desenvolvimento teórico do campo de pesquisa em gerenciamento de projetos. Trata-se de um desafio extremamente importante para a comunidade de pesquisadores (SAUER; REICH, 2007), pois o campo sofre tanto de escassa base teórica como de falta de conceitos (SHENHAR, 200I; SHENHAR; DVIR, 2004; AUBRY; HOBBS; THUILLIER, 2007). Para a prática, o modelo será uma referência útil para as empresas que estão considerando se devem ou não criar um EP-TI, ajudando-as a tomar decisões mais acertadas.

$\mathrm{Na}$ próxima seção, indicamos as bases teóricas do modelo proposto. Nas duas seções seguintes, apresentamos a metodologia e os resultados dos quatro estudos de caso. Em seguida, construímos e discutimos o modelo conceitual e suas implicações. Concluímos o artigo apresentando as limitações desta pesquisa e indicações para pesquisa futura. 


\section{BASES TEÓRICAS}

\subsection{UMA PERSPECTIVA SOBRE ESCRITÓRIOS DE PROJETOS}

A literatura apresenta muitas definições diferentes de EP, mas todas têm em comum uma ênfase no papel de centralização exercido pelo EP no referente ao gerenciamento dos projetos (BERNSTEIN, 2000; RAD, 200I; PROJECT MANAGEMENT INSTITUTE, 2004; DESOUZA; EVARISTO, 2006; HOBBS; AUBRY, 2007; AUBRY; HOBBS; THUILLIER, 2007). É oportuno apresentar as principais funções desempenhadas pelo EP, pois elas esclarecem como este exerce o papel de centralizador mencionado nas definições. Conforme Hobbs e Aubry (2007), os cinco principais grupos de funções de um EP são os seguintes: a) monitoramento e controle do desempenho dos projetos; b) desenvolvimento de competências e metodologias de gerenciamento de projetos; c) gerenciamento de múltiplos projetos; d) gerenciamento estratégico; e) aprendizado organizacional.

A literatura não acadêmica tradicionalmente caracteriza os EP em três diferentes níveis de acordo com seu posicionamento dentro da estrutura organizacional, a saber: a) nível I, de projeto; b) nível 2, departamental; c) nível 3, corporativo (ENGLUND; GRAHAN; DINSMORE, 2003; CRAWFORD, 2002). Nosso foco nesta pesquisa são os EP estabelecidos dentro da área de TI, portanto estamos tratando de EP de nível 2 ou I.

O tema EP-TI situa-se na intersecção de vários temas dentro da administração de tecnologia de informação. Conforme sugerido por Aubry, Hobbs e Thuillier (2007, p. 328, tradução nossa), "[...] o EP é parte de uma rede complexa de relacionamentos que liga estratégia, projetos e estruturas [...]”. Além da evidente intersecção com o tema gerenciamento de projetos de TI, podemos enxergar ainda intersecções com os temas gestão do portfólio de TI, estrutura organizacional de TI e governança de TI. No tema gerenciamento de projetos, trabalhos sobre o sucesso em gerenciamento de projetos (DELONE; MCLEAN, I992; MUNNS; BJEIRNI, I996; BACCARINI, I999; COOKE-DAVIES, 2002; JUGDEV; MÜLLER, 2005) são relevantes para o estudo de EP-TI, visto que um dos objetivos frequentemente buscado, quando se cria um EP, é aperfeiçoar o gerenciamento de projetos e reduzir o número de projetos problemáticos. Em especial, são relevantes estudos que pretendem avaliar o impacto do uso de processos padronizados e formais sobre o sucesso no gerenciamento dos projetos (JIANG; KLEIN; PICK, 2003; MILOSEVIC; PATANAKUL, 2005), uma vez que EP atuam no desenvolvimento de metodologias de gerenciamento de projetos. Por sua vez, o tema gestão de portfólio (MELLONE; WHARTON, I984; ELONEN; ARTTO, 2003; BLOMQUIST; MÜLLER, 2006) também é relevante para a pes- 
quisa sobre EP-TI, visto que um EP pode dar apoio ao gerenciamento estratégico de TI e gerenciar múltiplos projetos. Já no tema estrutura organizacional de TI, estudos relacionando a estrutura da área de TI com sua efetividade (BLANTON; WATSON; MOODY, I992; CASH; MCFARLAN; MCKENNEY, I992) também são relevantes para o estudo de EP-TI, pois um EP altera a estrutura da área de TI e pode aumentar sua efetividade na entrega de projetos. Finalmente, no tema governança de TI, também existe uma intersecção com o tema EP, uma vez que os padrões de autoridade de decisões situadas na esfera de atividades relacionadas ao gerenciamento de projetos (SAMBAMURTHY; ZMUD, I999; WEILL; ROSS, 2004) podem ser alterados por um EP.

Essa exposição não tem a intenção de ser uma revisão completa dos temas mencionados, mas apenas de apresentar evidências da importância do tema EP-TI dentro da administração de TI. Não obstante, a pesquisa sobre EP é relativamente escassa na literatura acadêmica, embora seja farta na literatura gerencial (AUBRY; HOBBS; THUILLIER, 2007), em que o tema EP tem sido frequentemente abordado do ponto de vista de promover o conceito e oferecer um guia a empresas interessadas em criar um EP (CRAWFORD, 2002; KERZNER, 2002; ENGLUND; GRAHAN; DINSMORE, 2003). Na literatura acadêmica, há pesquisas voltadas a avaliar a contribuição de um EP no sucesso dos projetos (DAI; WELLS, 2004; LIU; YETTON, 2007) e descrever as características de EP (DESOUZA; EVARISTO, 2006; HOBBS; AUBRY, 2007) e os processos e fatores críticos de sucesso da criação ou atuação do EP (DESOUZA; EVARISTO, 2006; MAXIMIANO; ANSELMO, 2006).

Nesta pesquisa, nosso interesse é entender quais são os fatores contextuais que direcionam a decisão de criar ou não criar um EP. O estudo contextual em gerenciamento de projetos tem recebido atenção dos pesquisadores que consideram que a abordagem tradicional no campo, denominada one-size-fits-all, não é adequada à realidade da prática de gerenciamento de projetos (SHENHAR, 200I; LIU; YETTON, 2007). Na revisão de literatura, não encontramos pesquisa acadêmica empírica sobre nosso tema de interesse. Uma exceção é Dai e Wells (2004), que apresentam dados empíricos descritivos para as motivações da criação de um EP. Porém, esses autores não se aprofundam sobre esse aspecto do tema EP. Logo, esta pesquisa contribui para o desenvolvimento do campo de pesquisa sobre EP preenchendo uma lacuna (WEBSTER; WATSON, 2002; KNOPF, 2006) no conhecimento sobre os fatores contextuais que direcionam a decisão de criar um EP-TI. 


\subsection{RAZÕES PARA IMPLEMENTAR OU NÃO UM EP}

A literatura apresenta diversas razões tanto para criar como para não criar um EP, conforme mostrado a seguir.

I. Razões para implementar um EP:

- Aperfeiçoar o gerenciamento de projetos e reduzir o número de projetos problemáticos: Bernstein (2000); Rad (200I); Englund, Grahan e Dinsmore (2003); Dai e Wells (2004); Desouza e Evaristo (2006); Maximiano e Anselmo (2006); Liu e Yetton (2007).

- Usar recursos de modo mais eficiente em ambiente de múltiplos projetos e melhorar a qualidade e satisfação do cliente: Dai e Wells (2004).

- Há necessidade de implementar projetos estratégicos: Block e Frame (1998); Bernstein (2000); Dai e Wells (2004).

- Facilitar a transferência de conhecimento sobre gerenciamento de projetos na organização: Bernstein (2000); Desouza e Evaristo (2006).

- Atenção às práticas de gerenciamento de projetos consideradas como as melhores pelo mercado: IT Governance Institute (2005).

2. Razões para não implementar um EP:

- Não há provas de que melhora o desempenho dos projetos: Englund, Grahan e Dinsmore (2003); Dai e Wells (2004).

- Aumenta a burocracia: Bernstein (2000); Englund, Grahan e Dinsmore (2003).

- Aumenta o custo fixo e pode não proporcionar benefícios que justificam esse aumento; estimula lutas de poder dentro da organização; surgem conflitos e ressentimentos com os gerentes de projetos; há perda de talentos para o gerenciamento de projetos; o ambiente é estável, sem importantes projetos a implementar; os métodos de gerenciamento de projetos e os resultados obtidos são satisfatórios: Englund, Grahan e Dinsmore (2003).

A extensa lista de razões tanto para criar como para não criar a entidade mostra que a criação de um EP pode ser controversa e gerar dúvidas na direção da empresa. Para cada razão encontrada na literatura para a criação de PMO, exceto uma delas, foi encontrada também uma razão em contrário. Por exemplo, contra a ideia de que um EP aperfeiçoa o gerenciamento de projetos e reduz o número de projetos problemáticos, encontrou-se a ideia de que não há provas de que um EP melhora o desempenho dos projetos. Da mesma forma, contra a ideia de que um EP promove o uso de recursos de modo mais eficiente em ambiente 
de múltiplos projetos, encontrou-se a ideia de que ele aumenta a burocracia e o custo fixo sem oferecer benefícios compatíveis. Cabe ressaltar, entretanto, que há apoio mais numeroso de autores para criar do que para não criar um EP. Porém, apenas Dai e Wells (2004) apresentam dados empíricos dos motivadores para a criação de EP, sem, contudo, fazerem referência às razões para não criá-lo. Em resumo, nota-se que a literatura carece de um estudo empírico que consolide as razões para criar e não criar um EP em uma visão única e integrada, que explique as circunstâncias em que a criação de um EP é mais indicada e aquelas em que essa criação é menos indicada.

Diante dessa situação, é evidente que executivos confrontados com uma proposta de criação de EP-IT em suas empresas não terão um apoio na literatura para tomar uma decisão bem fundamentada. Entretanto, pesquisadores interessados no assunto podem apresentar dois questionamentos:

- Se EP são tão benéficos, então por que tantas empresas ainda não tomaram a iniciativa de criar um?

- Se EP não apresentam resultados que justifiquem sua existência, então por que há uma tendência de criá-los nas empresas?

O fato de os mesmos autores que apresentam razões para criar o EP oferecerem também razões para não criar sugere que há, por um lado, condições organizacionais em que a criação de um EP se justifica claramente e, por outro, condições em que a criação do EP é difícil de ser justificada. O desafio, então, consiste em entender e sistematizar quais são essas condições, o que permitiria responder aos questionamentos apresentados anteriormente, bem como facilitaria o processo decisório do executivo que deve decidir pela criação ou não de um EP-IT. A seguir, enfrentamos esse desafio.

\section{MÉTODOS DE PESQUISA}

Embora a literatura apresente uma lista extensa de motivadores para a decisão de criação de EP, consideramos necessário realizar alguns estudos de caso para verificar a abrangência dessa lista e, se necessário, ampliá-la. O modelo conceitual será construído indutivamente com base em uma síntese dos motivadores relatados na literatura e encontrados nos estudos de caso.

Para garantir uma aplicação rigorosa do método de estudo de caso, seguimos as recomendações de Eisenhardt (I989) e Dubé e Paré (2003). Os casos foram selecionados entre empresas de grande porte, privadas, controladas por 
brasileiros e não orientadas a projetos. Essa escolha deve-se à opção de desenvolver teoria com escopo restrito, tendo em vista que

[...] elas não são confundidas e embaralhadas por terem de lidar com uma miríade de variáveis. Simplesmente, é mais fácil estudar fenômenos e testar teorias em domínios restritos (SAUER; REICH, 2007, p. 2).

Cumpre notar que nosso objetivo foi focar o contexto em que a decisão que nos interessa estudar parece ser mais difícil para os administradores. De fato, parece-nos que a decisão sobre a criação de um EP-TI nas empresas orientadas a projetos gera menor dúvida, pois a maior parte das receitas nessas empresas é obtida por meio de projetos. Ademais, excluímos as empresas de médio ou pequeno porte porque supomos que dificilmente a estrutura da área de TI nesses contextos pode comportar um EP-TI.

Definida a população de interesse, procuramos incluir em nossa amostra tanto empresas que têm um EP-TI como aquela que não têm. Então, selecionamos seis empresas nas quais seria provável ter acesso aos gestores de TI. Após sucessivos contatos com os administradores dessas empresas, conseguimos entrevistar executivos de quatro empresas, três das quais tinham um EP-TI (organizações A, B e D) e uma que não tinha (organização C).

Ao tratar da questão do número de casos necessários para gerar teoria, Eisenhardt (I989) afirma que de 4 a io casos usualmente são suficientes para obter saturação teórica, ponto em que a adição de novos casos não acrescenta elementos novos no entendimento do pesquisador sobre o fenômeno estudado. Portanto, não é possível afirmar que esse estágio foi alcançado, sobretudo no caso de empresas que não têm EP-TI. Essa limitação impacta os resultados da pesquisa no sentido de não nos permitir afirmar com segurança que a lista de motivadores que consideramos para a criação do modelo é a mais abrangente dentro da população de interesse.

O método de coleta de dados utilizado foi a condução de entrevistas com pessoas que atuam no EP-TI ou são gerentes funcionais do departamento de TI. Entrevistamos mais de uma pessoa em cada organização com o intuito de obter triangulação na coleta de dados. Ao final, entrevistamos io pessoas, conforme indicado na Tabela I. Nas empresas com EP-TI, além de entrevistarmos um funcionário que atua no EP-TI, logramos entrevistar também pelo menos um executivo. 


\section{TABELA I}

PESSOAS ENTREVISTADAS EM CADA ORGANIZAÇ ÃO

\begin{tabular}{lccc}
\hline & ATUAM NO EP-TI & $\begin{array}{c}\text { GERENTE DE APOIO } \\
\text { (STAFF) EM TI }\end{array}$ & $\begin{array}{c}\text { GERENTES FUNCIONAIS } \\
\text { EM TI }\end{array}$ \\
\hline Organização A & 1 & 1 & - \\
\hline Organização B & 1 & 1 & 2 \\
\hline Organização C & - & 1 & 2 \\
\hline Organização D & 1 & - & - \\
\hline
\end{tabular}

Fonte: Elaborada pelos autores.

Um protocolo de entrevistas foi preparado e enviado aos entrevistados previamente. Algumas das questões eram abertas, outras tinham o formato de múltipla escolha. Tínhamos dois propósitos com as questões: I. obter um quadro claro do contexto do departamento de TI, do EP-TI e do gerenciamento de projetos de TI; e 2. entender as motivações para o estabelecimento do EP-TI ou, alternativamente, as motivações para a decisão de não criar um.

As entrevistas foram realizadas nos escritórios das empresas e gravadas, transcritas, sintetizadas e enviadas aos entrevistados para validação. Após a validação, as transcrições das entrevistas foram examinadas por meio de técnicas de análise de conteúdo (TROCHIM; DONNELLY, 2007). Basicamente, procuramos, ao longo do texto, os trechos em que os entrevistados explicam os motivos para a decisão sobre a criação do EP, bem como o que se esperava como resultado dessa decisão. Posteriormente, categorizamos esses trechos criando uma lista dos motivadores encontrados nos estudos de caso. Finalmente, sintetizamos os motivadores descritos na literatura e nos estudos de caso, e construímos o modelo conceitual, que é o objetivo desta pesquisa. A Figura i exibe uma visão gráfica do método adotado para gerar o modelo conceitual. 


\section{FigurA I}

\section{MÉTODO ADOTADO PARA GERAR O MODELO CONCEITUAL}

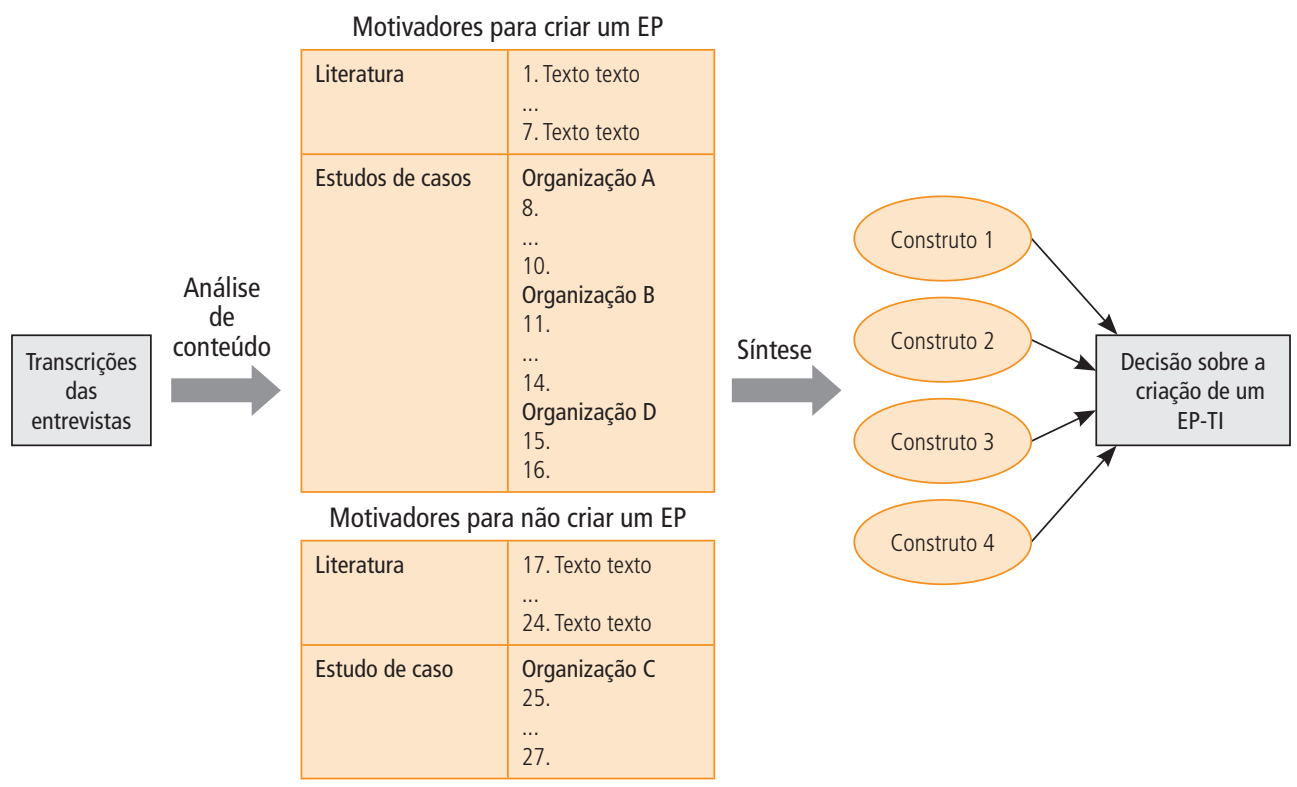

Fonte: Elaborada pelos autores.

\section{RESULTADOS}

A Tabela 2 apresenta um sumário das características das organizações estudadas. Todas são grandes empresas privadas brasileiras, não orientadas a projetos e com operações internacionais. Há uma grande variação em seus tamanhos, pois uma delas é uma das maiores corporações brasileiras. A organização $\mathrm{C}$ tem o maior número de projetos de TI em andamento, com enorme distância das demais, o que não é uma surpresa, pois TI tem um papel crítico no setor de serviços financeiros. Contudo, é importante destacar que não é possível comparar diretamente os números de projetos, pois cada organização usa diferentes critérios para determinar o que é considerado um projeto. A organização B tem a maior taxa de sucesso em projetos e, talvez por coincidência, possui o mais antigo EP-TI em operação. Foram omitidos dados que pudessem revelar a identidade das organizações (indicados na Tabela 2 com asteriscos). 


\section{TABELA 2}

SUMÁRIO DAS CARACTERÍSTICAS DAS ORGANIZAÇÖES ESTUDADAS (BASE 31.12.2006)

\begin{tabular}{|c|c|c|c|c|}
\hline & $\begin{array}{c}\text { ORGANIZAÇÃO } \\
\text { A }\end{array}$ & $\begin{array}{c}\text { ORGANIZAÇÃO } \\
\text { B }\end{array}$ & $\begin{array}{c}\text { ORGANIZAÇÃO } \\
\text { C }\end{array}$ & $\begin{array}{c}\text { ORGANIZAÇÃO } \\
\text { D }\end{array}$ \\
\hline Setor & $\begin{array}{c}\text { Indústria (bens } \\
\text { de consumo) }\end{array}$ & $* \star *$ & $\begin{array}{l}\text { Serviços } \\
\text { financeiros }\end{array}$ & $\star * *$ \\
\hline $\begin{array}{l}\text { Número de funcionários } \\
\text { (FTE) }\end{array}$ & $\begin{array}{c}\text { Cerca de } \\
5.000\end{array}$ & $\begin{array}{c}\text { Cerca de } \\
38.000\end{array}$ & $\star \star \star *$ & $\begin{array}{c}\text { Cerca de } \\
19.000\end{array}$ \\
\hline Faturamento bruto & US\$ $1,8 \mathrm{bi}$ & US $\$ 16,9$ bi & $* \star *$ & US\$ 4,4 bi \\
\hline $\begin{array}{l}\text { Número médio de } \\
\text { projetos em andamento }\end{array}$ & 35 & 250 & $\begin{array}{l}\text { Cerca de } \\
1.500\end{array}$ & 22 \\
\hline $\begin{array}{l}\text { Percentual de projetos } \\
\text { concluídos dentro do } \\
\text { prazo, custo e qualidade }\end{array}$ & $50 \%$ & $73 \%$ & Não disponível & $67 \%$ \\
\hline Ano de criação do EP-TI & 2006 & 2002 & Não há EP-TI & 2007 \\
\hline
\end{tabular}

Fonte: Elaborada pelos autores.

A decisão de criação do EP-TI na organização A foi tomada durante uma reestruturação organizacional em TI, visando criar uma estrutura mais enxuta e voltada ao negócio. Constatamos que os motivadores para a criação do EP-TI nessa organização foram os seguintes: a) pouca satisfação com a capacidade de entrega de projetos de TI, b) pouca satisfação com as práticas e os métodos de gestão de projetos e gestão do conhecimento a respeito, c) necessidade de sustentar a estratégia de crescimento da organização. O Quadro I mostra um trecho selecionado da entrevista e os motivadores identificados. 


\section{QUADRO I}

\section{AMOSTRA DA ANÁLISE DE CONTEÚDO}

REALIZADA NA ORGANIZAÇÃO A

\begin{tabular}{|c|c|}
\hline MOTIVADOR & TRECHO DA ENTREVISTA \\
\hline & $\begin{array}{l}\text { Pesquisador: "O que eu gostaria de saber, quais foram as razões } \\
\text { que justificaram a criação do escritório de projetos. Eu gostaria que } \\
\text { você me contasse um pouco da história, em que momento, que } \\
\text { contexto organizacional a [...] estava passando no momento da } \\
\text { criação...?" }\end{array}$ \\
\hline $\begin{array}{l}\text { Necessidade de } \\
\text { sustentar a estratégia } \\
\text { de crescimento da } \\
\text { organização }\end{array}$ & $\begin{array}{l}\text { Resp. Conhecimento e Controle: "[...] a empresa vai passar agora } \\
\text { por um momento de crescimento, a estratégia da empresa está } \\
\text { voltada à expansão, principalmente à expansão internacional } \\
\text { [...] o ambiente de sistemas da operação Brasil, ele precisa ser } \\
\text { totalmente reestruturado, reformulado, e isso vai demandar }\end{array}$ \\
\hline $\begin{array}{l}\text { Pouca satisfação com a } \\
\text { capacidade de entrega } \\
\text { de projetos de TI }\end{array}$ & $\begin{array}{l}\text { fortes investimentos em TI nos próximos anos. [....] e isso também } \\
\text { junta-se com o fator de que historicamente não tínhamos uma } \\
\text { efetividade em realização de projetos, a entrega de projetos era } \\
\text { menor do que o planejado, com desvios de prazo, com desvios de } \\
\text { custo, com desvios de qualidade, então o escritório de projetos, } \\
\text { ele, além de atender a essa estratégia de crescimento, ele vem } \\
\text { com uma missão forte aí de melhorar a efetividade, de qualidade } \\
\text { na entrega dos produtos." }\end{array}$ \\
\hline
\end{tabular}

Fonte: Elaborado pelos autores.

A organização B possui dois EP-TI. O primeiro foi criado como consequência natural da adoção de uma estrutura matricial e criação de uma gerência de projetos em TI. O segundo foi criado anos mais tarde para acompanhar e relatar para a direção de TI a situação de um portfólio de projetos estratégicos. Em ambos os momentos, a área de TI passava por reestruturações para dar mais agilidade à área, de modo que ela pudesse suportar mais eficazmente as estratégias de negócios. Nessa organização, constatamos que os motivadores para a criação do EP-TI foram os seguintes: a) pouca satisfação com a metodologia e as práticas de gerenciamento de projetos (primeiro EP), b) pouca satisfação com o cumprimento de prazos (primeiro EP), c) contato com as melhores práticas de mercado (primeiro EP), d) pouca satisfação com o controle do portfólio estratégico de TI (segundo EP). O Quadro 2 mostra um trecho selecionado da entrevista e o motivador identificado. 


\section{QUADRO 2}

\section{AMOSTRA DA ANÁLISE DE CONTEÚDO REALIZADA NA ORGANIZAÇÃO B}

\begin{tabular}{|c|c|}
\hline MOTIVADOR & TRECHO DA ENTREVISTA \\
\hline $\begin{array}{l}\text { Pouca satisfação com } \\
\text { o controle do portfólio } \\
\text { estratégico de TI } \\
\text { (segundo EP) }\end{array}$ & $\begin{array}{l}\text { Pesquisador: "[...] falávamos das razões que justificaram a criação } \\
\text { do escritório de projetos..." } \\
\text { Responsável EP: "[...] É muita coisa [projetos estratégicos } \\
\text { voltados ao crescimento], é muito grande, [o ClO] não conseguia } \\
\text { acompanhar, então eu vou separar alguns que a gente não pode } \\
\text { falhar, nesses eu vou ter um acompanhamento mais de perto... } \\
\text { o [primeiro EP] porque houve o momento [de reestruturação], o } \\
\text { [segundo EP] foi mais pensando nesse momento de crescimento, } \\
\text { é muito mais investimento que estava sendo feito, está crescendo } \\
\text { muito a demanda com mais projetos, então tinha que ter uma } \\
\text { estrutura para ajudar a ter o acompanhamento desse controle." }\end{array}$ \\
\hline
\end{tabular}

Fonte: Elaborado pelos autores.

Muito embora a organização C seja uma das maiores corporações brasileiras e invista pesadamente em TI, pois atua no segmento financeiro, ela não possui EP-TI. Constatamos que os motivadores da decisão de não criar um EP-TI nessa organização são os seguintes: a) satisfação com o desempenho em gerenciamento de projetos e com o controle do portfólio; b) opinião de que um EP estimula a burocracia, aumenta o custo fixo e pode desviar a atenção da entrega dos projetos; c) opinião de que não há evidências concretas de que um EP aumenta o sucesso dos projetos. O Quadro 3 mostra um trecho selecionado da entrevista e o motivador identificado.

\section{QUADRO 3}

\section{AMOSTRA DA ANÁLISE DE CONTEÚDO REALIZADA NA} ORGANIZAÇÃO C

\begin{tabular}{ll}
\hline MOTIVADOR & TRECHO DA ENTREVISTA \\
\hline & $\begin{array}{l}\text { Pesquisador: "Essa informalidade [na metodologia], vocês } \\
\text { consideram como positivo porque dá flexibilidade, agilidade, não }\end{array}$ \\
$\begin{array}{l}\text { Um EP aumenta a } \\
\text { burocracia, aumenta o } \\
\text { custo fixo e pode desviar }\end{array}$ & $\begin{array}{l}\text { Responsável Governança de TI: “[...] quando você começa a criar } \\
\text { a atenção da entrega }\end{array}$ \\
$\begin{array}{l}\text { dos projetos } \\
\text { a buitos documentos, muito formalismo, você aumenta muito e existe muita dúvida de quanto vale a pena... o } \\
\text { que você tem de benefício com isso?... se você consegue ganhar } \\
\text { alguma coisa no final da linha, não é muito claro [...]." }\end{array}$ \\
\hline
\end{tabular}




\section{QUADRO 3 (CONTINUAÇÃO)}

\section{AMOSTRA DA ANÁLISE DE CONTEÚDO REALIZADA NA ORGANIZAÇÃO C}

\begin{tabular}{ll}
\hline MOTIVADOR & TRECHO DA ENTREVISTA \\
\hline & $\begin{array}{l}\text { Pesquisador: " [concordam que um EP] aumenta o custo fixo, } \\
\text { pode não valer a pena, estimula a burocracia, [desviar a atenção } \\
\text { da entrega dos projetos]...?" }\end{array}$ \\
$\begin{array}{l}\text { Um EP aumenta a } \\
\text { burocracia, aumenta o } \\
\text { custo fixo e pode desviar } \\
\text { a atenção da entrega } \\
\text { dos projetos }\end{array}$ & $\begin{array}{l}\text { tem mais gente gerenciando do que fazendo gol ... pode distrair } \\
\text { da entrega de projetos, eu acho que isso também é um risco, } \\
\text { porque você começa a olhar tanto o meio, é tão mais importante } \\
\text { a forma do que o conteúdo." } \\
\text { Responsável Infraestrutura: "Acho que aqui pode sim, aí pode } \\
\\
\text { ser..." }\end{array}$ \\
\hline
\end{tabular}

Fonte: Elaborado pelos autores.

A organização D vem experimentando diferentes abordagens para aperfeiçoar suas competências em gerenciamento de projetos na área de TI, o que incluiu a adoção de uma estrutura matricial e a criação de uma gerência de projetos em TI, bem como a criação de EP de níveis I e 2. Nessa organização, constatamos que os motivadores das decisões de criar os EP-TI foram os seguintes: a) insatisfação com a metodologia de gerenciamento de projetos no referente ao estabelecimento de requisitos, b) insatisfação com a capacidade de TI de entregar projetos dentro do prazo, custo e com a qualidade esperada. O Quadro 4 mostra um trecho selecionado da entrevista e o motivador identificado.

\section{QUADRO 4}

AMOSTRA DA ANÁLISE DE CONTEÚDO REALIZADA NA ORGANIZAÇÃO D

\section{MOTIVADOR TRECHO DA ENTREVISTA}

Pesquisador: "O que você sente como mais importante para você, Insatisfação com a capacidade de TI de entregar projetos dentro do prazo, custo e com a qualidade esperada enfim, quando te perguntarem, isso aí [EP de nível 2] realmente vai gerar custo fixo para você, e aí a sua liderança vai dizer, mais custo fixo? Como é que você vai defender [o EP de nível 2] ?" CIO: "Como eu defendo isso, eu consigo rastrear e garantir, ter melhor garantia de que o projeto vai ser cumprido no prazo, no escopo e no custo [...]." 
O Quadro 5 apresenta um resumo dos motivadores para criar um EP que foram encontrados na literatura e nos estudos de caso. Podemos notar uma grande similaridade entre os motivadores para criação de EP-TI encontrados na literatura e nos estudos de casos. Por exemplo, o motivador I encontra correspondência com os motivadores 7, 8, IO, II, I4 e I5. O motivador 3, com os motivadores 7, II e I5. O motivador 4, com o motivador 9 . O motivador 5 , com o motivador 8. O motivador 6, com o motivador I2. Dos motivadores encontrados na literatura, apenas o motivador 2 não foi confirmado pelos estudos de caso. Na relação inversa, entre os motivadores encontrados nos estudos de caso, o motivador I3 não é mencionado na literatura. Assim, os estudos de caso serviram para confirmar e enriquecer os motivadores para criar um EP relatados pela literatura.

\section{QUADRO 5}

\section{RESUMO DOS MOTIVADORES PARA CRIAR UM EP}

\section{MOTIVADORES PARA CRIAR UM EP}

1. Aperfeiçoar o gerenciamento de projetos e reduzir o número de projetos problemáticos.

2. Usar recursos de modo mais eficiente em ambiente de múltiplos projetos.

3. Melhorar a qualidade e a satisfação do cliente.

Literatura 4. Há necessidade de implementar projetos estratégicos.

5. Facilitar a transferência de conhecimento sobre gerenciamento de projetos na organização.

6. Atenção às práticas de gerenciamento de projetos consideradas como as melhores pelo mercado.

Organização A:

7. Pouca satisfação com a capacidade de entrega de projetos de TI.

8. Pouca satisfação com as práticas e os métodos de gestão de projetos e gestão do conhecimento a respeito.

9. Necessidade de sustentar a estratégia de crescimento da organização.

Organização B:

Estudos projetos.

de caso 11. Pouca satisfação com o cumprimento de prazos.

12. Contato com as melhores práticas de mercado.

13. Pouca satisfação com o controle do portfólio estratégico de TI.

Organização D:

14. Insatisfação com a metodologia de gerenciamento de projetos em geral e no referente ao estabelecimento de requisitos.

15. Insatisfação com a capacidade de TI de entregar projetos dentro do prazo, custo e com a qualidade esperada. 
O Quadro 6, por sua vez, apresenta uma síntese dos motivadores para não criar um EP que foram encontrados na literatura e nos estudos de caso. Podemos notar também uma grande similaridade entre os motivadores para não criar um EP-TI encontrados na literatura e nos estudos de casos. Por exemplo, o motivador 16 encontra correspondência com o motivador 26. Os motivadores I7 e I8, com o motivador 25. O motivador 23, com o motivador 24. Dos motivadores encontrados na literatura, os motivadores I9, 20, 21 e 22 não foram confirmados pelos estudos de caso. Na relação inversa, todos os motivadores encontrados nos estudos de caso foram mencionados na literatura. Assim, o único estudo de caso de empresas que não têm EP-TI serviu para confirmar alguns dos motivadores relatados pela literatura para não criar um EP, mas não para ampliar a lista de motivadores. Essa é uma limitação da pesquisa, cujos impactos no modelo final não podem ser avaliados sem que mais pesquisas sejam realizadas.

\section{QUADRO 6}

\section{SÍNTESE DOS MOTIVADORES PARA NÃO CRIAR UM EP}

\section{MOTIVADORES PARA NÃO CRIAR UM EP}

16. Não há provas de que melhora o desempenho dos projetos.

17. Aumenta o custo fixo e pode não proporcionar benefícios que justificam esse aumento.

18. Aumenta a burocracia.

Literatura

19. Estimula lutas de poder dentro da organização.

20. Surgem conflitos e ressentimentos com os gerentes de projetos.

21. Perda de talentos para o gerenciamento de projetos.

22. O ambiente é estável, sem importantes projetos a implementar.

23. Os métodos de gerenciamento de projetos e os resultados obtidos são satisfatórios.

\section{Organização C}

24. Satisfação com o desempenho em gerenciamento de projetos e com o

Estudo controle do portfólio.

de caso 25. Um EP estimula a burocracia, aumenta o custo fixo e pode desviar a atenção da entrega dos projetos.

26. Não há evidências concretas de que aumenta o sucesso dos projetos. 


\section{CONSTRUÇÃO DO MODELO CONCEITUAL}

Nosso próximo passo é criar um modelo conceitual para a decisão de criação de um EP-TI. Para tanto, vamos "[...] explicar quais conceitos e argumentos causais são adotados das fontes citadas e como eles são vinculados à teoria que está sendo desenvolvida" (SUTTON; STAW, I995, p. 373, tradução nossa). Desejamos que esse modelo seja parcimonioso na inclusão de motivadores, mas que, ao mesmo tempo, seja abrangente o bastante para abrigar todos os aspectos dessa decisão (HAIR et al., 2003; WHETTEN, I989). Vale recordar que a Figura I exibe uma visão geral do método adotado.

Analisando os quadros 5 e 6 , percebemos que há motivadores que expressam opiniões sobre os resultados, bons ou ruins, provocados pela criação de um EP-TI. Podemos incluir nessa categoria os motivadores de I a 3, 5, 6, I2, de I6 a 2I, 25 e 26 . Os demais motivadores (4, de 7 a II, de I3 a I5, de 22 a 24) expressam o reconhecimento de uma situação, confortável ou desconfortável.

Enfocando os motivadores que expressam opiniões e sintetizando-os, podemos extrair o primeiro construto de nosso modelo: opinião sobre EP-TI. Cabe notar que os motivadores de I a 3, 5, 6 e I2 manifestam uma opinião favorável, ao passo que os motivadores de I6 a 2I, 25 e 26 expressam uma opinião desfavorável.

Enfocando agora os motivadores que expressam o reconhecimento de uma situação, notamos que existem semelhanças evidentes entre eles. Por exemplo, os motivadores 7, 8, IO, II, I4, I5, 23 e 24 (parte dele) apresentam o reconhecimento de uma situação de satisfação (23 e 24) ou de insatisfação (demais) com relação à entrega dos projetos e metodologia de gerenciamento. Assim, vamos obter nosso segundo construto sintetizando esses motivadores conforme segue: satisfação com a entrega dos projetos de TI.

Restaram os motivadores 4, 9, I3 e 22. Uma análise rápida aponta para uma forte proximidade entre os motivadores 4 , 9 e 22. Neles, há referências à importância estratégica, ou à falta dela, dos projetos que devem ser executados. Assim, obtemos nosso terceiro construto resumindo esses motivadores conforme segue: importância estratégica do portfólio de projetos de TI.

Finalmente, restaram-nos os motivadores i3 e 24 (parte dele), que faz referência à satisfação com o controle do portfólio projetos de TI, que se torna assim nosso último construto.

Para facilidade de visualização, apresentamos, a seguir, a síntese dos motivadores encontrados na literatura e nos estudos de casos que gerou os quatro construtos presentes no modelo conceitual: 
a) Construto opinião sobre EP-TI:

I. Aperfeiçoar o gerenciamento de projetos e reduzir o número de projetos problemáticos.

2. Usar recursos de modo mais eficiente em ambiente de múltiplos projetos.

3. Melhorar a qualidade e a satisfação do cliente.

5. Facilitar a transferência de conhecimento sobre gerenciamento de projetos na organização.

6. Atenção às práticas de gerenciamento de projetos consideradas como as melhores pelo mercado.

I2. Contato com as melhores práticas de mercado.

I6. Não há provas de que melhora o desempenho dos projetos.

I7. Aumenta o custo fixo e pode não proporcionar benefícios que justificam esse aumento.

I8. Aumenta a burocracia.

I9. Estimula lutas de poder dentro da organização.

20. Surgem conflitos e ressentimentos com os gerentes de projetos.

2I. Perda de talentos para o gerenciamento de projetos.

25. Um EP estimula a burocracia, aumenta o custo fixo e pode desviar a atenção da entrega dos projetos.

26. Não há evidências concretas de que aumenta o sucesso dos projetos.

b) Construto satisfação com entrega dos projetos de TI:

7. Pouca satisfação com a capacidade de entrega de projetos de TI.

8. Pouca satisfação com as práticas e os métodos de gestão de projetos e gestão do conhecimento a respeito.

Io. Pouca satisfação com a metodologia e as práticas de gerenciamento de projetos.

II. Pouca satisfação com o cumprimento de prazos.

I4. Insatisfação com a metodologia de gerenciamento de projetos em geral e no referente ao estabelecimento de requisitos.

I5. Insatisfação com a capacidade de TI de entregar projetos dentro do prazo, custo e com a qualidade esperada.

23. Os métodos de gerenciamento de projetos e os resultados obtidos são satisfatórios.

24. Satisfação com o desempenho em gerenciamento de projetos.

c) Construto importância estratégica do porftólio de projetos de TI:

4. Há necessidade de implementar projetos estratégicos.

9. Necessidade de sustentar a estratégia de crescimento da organização.

22. O ambiente é estável, sem importantes projetos a implementar. 
d) Construto satisfação com o controle do porftólio de projetos de TI:

I3. Pouca satisfação com o controle do portfólio estratégico de TI.

24. Satisfação com o desempenho em controle do portfólio.

A Figura 2 exibe o modelo conceitual obtido. Por simplicidade, excluímos da expressão dos construtos os termos "de TI" e "de projetos de TI", que ficam subentendidos, uma vez que estamos tratando de uma decisão de criação de escritório de projetos na área de TI. Cumpre notar que o modelo proposto aplica-se a grandes empresas brasileiras, privadas, não orientadas a projetos, pois foi com esse contexto em mente que desenvolvemos o modelo.

\section{FIGURA 2}

MODELO CONCEITUAL DOS DIRECIONADORES DA DECISÃO DE CRIAÇÃO DE UM EP-TI

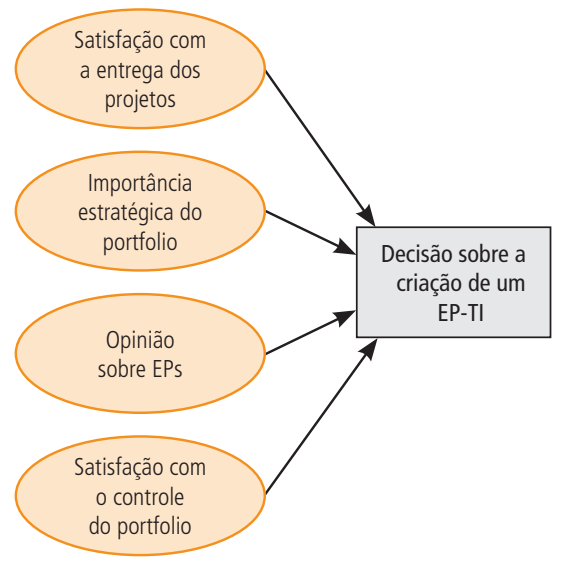

Fonte: Elaborada pelos autores.

\section{DISCUSSÃO E IMPLICAÇÕES}

Vamos agora analisar "[...] quais são as dinâmicas psicológicas, econômicas ou sociais subjacentes que justificam a seleção dos fatores e as relações de causalidade propostas" (WHETTEN, I989, p. 49I, tradução nossa). Conforme enfatizado por Webster e Watson (2002, p. XIX, tradução nossa), “[...] o raciocínio ou a justificativa para essas relações representa a parte crucial do processo de desenvolvimento de teoria".

A criação formal de um EP-TI implica a criação de custo fixo, por menor que ele seja. Portanto, a ideia de criar um EP-TI é analisada com cuidado, tal- 
vez mesmo com ceticismo, pelos executivos que tomam a decisão. Quais fatores terão peso nessa decisão? É lícito supor que serão considerados tanto fatores mais objetivos ligados ao contexto da empresa como fatores mais subjetivos ligados às percepções dos indivíduos sobre a natureza de EP e os resultados que ele proporciona. O conjunto desses fatores determinará a decisão sobre a criação de um EP-TI.

No modelo mostrado na Figura I, essa dinâmica está representada por quatro construtos latentes ligados a uma variável dependente, observável, dicotômica ( $\operatorname{sim} /$ não). Os fatores contextuais e mais objetivos ligados ao contexto da empresa e da área de TI estão representados pelos construtos satisfação com a entrega dos projetos, importância estratégica do portfólio e satisfação com o controle do portfólio. Os fatores mais subjetivos e ligados a percepções, experiências e preconceitos dos executivos sobre a entidade EP estão resumidos pelo construto opinião sobre EP.

Para ilustrar a dinâmica do modelo, vamos imaginar que uma empresa possua o seguinte contexto: a) baixo nível de satisfação com a entrega dos projetos de TI, por exemplo, devido a atrasos, b) há projetos estrategicamente importantes a executar na área de TI, c) a direção da empresa já ouviu falar sobre EP e acredita que sua criação pode ajudar a reduzir atrasos em projetos. Nesse contexto, é muito provável que a empresa decida criar um EP-TI. No entanto, se imaginarmos um outro contexto, em que há alto nível de satisfação com a entrega dos projetos de TI, então, mesmo que existam projetos estratégicos a executar e a direção tenha uma opinião favorável sobre EP-TI, é provável que a resistência à criação de custo fixo seja muito forte e a empresa decida não criar o EP-TI. Em síntese, esses exemplos ilustram o modo como o contexto da empresa é capturado pelos construtos, cuja ação combinada tende a conduzir a empresa para o sim ou para o não na decisão sobre criação de um EP-TI.

Uma parte importante no trabalho de desenvolvimento teórico é a identificação de hipóteses rivais, bem como a antecipação de evidências que mostrariam que a teoria está errada (KING; KEOHANE, I994). No referente a hipóteses rivais, podemos certamente imaginar modelos alternativos com mais ou menos construtos, ou com construtos definidos diferentemente, ou ainda com relacionamentos não indicados na Figura I. As possibilidades de modificar esse modelo teórico são inúmeras e podem servir como um "um guia útil para pesquisa" (WHETTEN, I989, p. 49I, tradução nossa). No referente à antecipação de evidências contra a validade do modelo, elas consistem em casos de empresas que contrariem a lógica do modelo anteriormente apresentada. Por exemplo, se existir uma empresa que decidiu criar um EP-TI, muito embora estivesse satisfeita com a entrega de seus projetos, estivesse satisfeita também com o controle de seu portfólio, não tivesse projetos estratégicos a implementar e, finalmente, 
a opinião da direção sobre EP fosse desfavorável, então esta será uma forte evidência contra a validade do modelo. Ou, vice-versa, se existir uma empresa que decidiu não criar um EP-TI, muito embora não estivesse satisfeita com a entrega de seus projetos, não estivesse satisfeita também com o controle de seu portfólio, tivesse projetos estratégicos a implementar e, finalmente, a opinião da direção sobre EP fosse favorável, então esta será outra forte evidência contra a validade do modelo.

O modelo proposto apresenta duas implicações interessantes. A primeira e mais óbvia é que ele pode servir como um apoio para executivos que estão considerando se devem ou não criar um EP-TI em suas empresas. Ao oferecer insights sobre o contexto das empresas que decidiram criar EP-TI, bem como das que decidiram não fazê-lo, o modelo pode estimular executivos a examinar o contexto de suas empresas com uma perspectiva mais ampla, auxiliando-os a tomar decisões mais acertadas. A segunda é que o modelo pode auxiliar a entender e prever como a evolução do contexto empresarial irá impactar a existência do EP-TI. Por exemplo, caso aumente a satisfação com as entregas dos projetos, diminua o número de projetos estratégicos e melhore o controle do portfólio, é lícito prever que a continuidade do EP-TI poderia ser questionada. Outra possibilidade é que esse questionamento ocorra por conta de uma mudança na direção da empresa, tendo a nova direção uma opinião pouco favorável em relação a EP. Nesse caso, a existência do EP-TI também poderia ser questionada. Portanto, o modelo pode servir como uma referência teórica para explicar por que muitas empresas estão abrindo EP-TI, enquanto outras não fazem isso, muito embora tenham uma gerência esclarecida no comando da área de TI. Pode servir também para explicar por que muitos EP “[...] estão sendo fechados ou radicalmente redesenhados quase tão rapidamente [como são criados]” (HOBBS; AUBRY, 2007, p. 79).

\subsection{LIMITAÇõES E SUGESTÕES PARA PESQUISA FUTURA}

O modelo conceitual exibido na Figura I possui duas limitações. A primeira refere-se à quantidade e às características dos casos estudados. De fato, o modelo foi construído com um pequeno número de casos, particularmente com relação a empresas que não têm EP-TI. No entanto, consideramos que o efeito dessa limitação sobre o modelo não é tão relevante, uma vez que ele foi construído também com base na literatura, que não enfoca contextos empresariais específicos. Visto que nenhum dos motivadores relatados na literatura foi descartado por não encontrar respaldo nos casos estudados, é lícito supor que o modelo é bastante abrangente e válido para empresas de qualquer setor, tanto para as que decidiram criar um EP-TI como para as que decidiram não fazê-lo. A segunda 
limitação refere-se à subjetividade inerente ao procedimento usado para sintetizar os motivadores e criar os construtos presentes no modelo. Portanto, é lícito supor que há modelos alternativos com relação ao número de construtos incluídos no modelo, bem como em relação às descrições dos construtos. Em outras palavras, outros pesquisadores que analisarem os mesmos dados poderão chegar a modelos um pouco diferentes, mas igualmente válidos logicamente e consistentes com os dados empíricos e a literatura. Não obstante, o modelo proposto será um ponto de partida útil para pesquisadores interessados no tema, o que nos leva às sugestões para pesquisa futura, que abordamos a seguir.

O modelo conceitual proposto - ou modelos alternativos - precisa ser testado empiricamente. Sugerimos que esse teste seja realizado por meio de modelagem com equações estruturais. Uma outra possibilidade de pesquisa futura é a aplicação de regressão logística ao modelo proposto, visando criar uma regra para discriminar empresas em que existe EP-TI daquelas em que ele não existe. Essa regra seria uma ferramenta útil para administradores, pois ofereceria evidências do grupo a que uma dada empresa tem maior probabilidade de pertencer, permitindo uma tomada de decisão mais bem fundamentada sobre a criação de EP-TI. Finalmente, a generalização do modelo para empresas de todos os tamanhos dentro de setores específicos - por exemplo, apenas empresas orientadas a projetos, ou apenas empresas do setor financeiro - também nos parece ser um tema interessante para pesquisa futura, permitindo a realização de interessantes estudos comparativos. Para superar a limitação encontrada nesta pesquisa quanto ao pequeno número de casos de empresas sem EP-TI, recomenda-se que nos estudos futuros a amostra contenha um número maior de casos dessa natureza.

\section{CONCLUSÃO}

Em razão do crescimento de investimentos em TI e da importância estratégica destes, muitas empresas decidiram criar um escritório de projetos na área de TI (EP-TI). Entretanto, muitas empresas optaram por não fazer isso. Este artigo apresenta um modelo conceitual da decisão de criação de um EP-TI que pretende explicar os porquês dessas decisões. Esse modelo propõe que a decisão depende de quatro direcionadores: a satisfação com a entrega dos projetos, a importância estratégica do portfólio, a satisfação com o controle do portfólio e a opinião sobre escritórios de projetos. O contexto da empresa determina a força e o sentido desses direcionadores; a soma de seus efeitos determina a decisão da empresa entre criar ou não criar um EP-TI.

O modelo proposto é um primeiro passo no entendimento das razões subjacentes à criação de escritórios de projetos fora das organizações orientadas a 
projetos e pode ser útil para executivos confrontados com a decisão de criar ou não criar um EP-TI. Entretanto, essa teoria ainda precisa ser testada empiricamente, e seu potencial para suportar decisões gerenciais ainda precisa ser plenamente explorado. Esperamos que outros pesquisadores sintam-se atraídos por esses desafios.

\section{REFERÊNCIAS}

AUBRY, M.; HOBBS, B.; THUILLIER, D. A new framework for understanding organisational project management through the PMO. International Journal of Project Management, v. 25, n. 4, p. 328-336, May 2007 .

BACCARINI, D. The logical framework method for defining project success. Project Management Journal, v. 30, n. 4, p. 25-32, Dec. I999.

BERNSTEIN, S. Project offices in practice. Project Management Journal, v. 3I, n. 4, p. 4-6, Dec. 2000 .

BLANTON, J. E.; WATSON, H. J.; MOODY, J. Toward a better understanding of information technology organization: a comparative case study. MIS Quarterly, v. I6, n. 4, p. 53I-555, Dec. I992. BLOCK, T.; FRAME, J. The project office. Menlo Park: Crisp Learning, I998.

BLOMQUIST, T.; MÜLLER, R. Practices, roles, and responsibilities of middle managers in program and portfolio management. Project Management Journal, v. 37, n. I, p. 52-66, Mar. 2006.

CASH JR., J. I.; MCFARLAN, F. W.; MCKENNEY, J. L. Corporate information systems management: the issues facing senior executives. Homewood: Richard D. Irwin, I992.

CRAWFORD, J. K. The strategic project office. Boca Raton: Taylor \& Francis, 2002.

COOKE-DAVIES, T. The real success factors on projects. International Journal of Project Management, v. 20, n. 3, p. I85-I90, Apr. 2002.

DAI, C. X.; WELLS, W. G. An exploration of project management office features and their relationship to project performance. International Journal of Project Management, v. 22, n. 7, p. 523-532, Oct. 2004 .

DELONE, W. H.; MCLEAN, E. R. Information systems success: the Quest for the dependent variable. Information Systems Research, v. 3, n. I, p. 60-95, Mar. I992.

DESOUZA, K. C.; EVARISTO, J. R. Project management offices: a case of knowledge-based archetypes. International Journal of Information Management, v. 26, n. 5, p. 4I4-423, Oct. 2006.

DUBÉ, L.; PARÉ, G. Rigor in information systems positivist case research: current practices, trends and recommendations. MIS Quarterly, v. 27, n. 4, p. 597-635, Dec. 2003.

EISENHARDT, K. M. Building theories from case study research. Academy of Management Review, v. I4, n. 4, p. 532-550, Oct. I989.

ELONEN, S.; ARTTO, K. A. Problems in managing internal development projects in multi-project environments. International Journal of Project Management, v. 21, n. 6, p. 395-402, Aug. 2003.

ENGLUND, R. L.; GRAHAM, R. J.; DINSMORE, P. C. Creating the project office - a manager's guide to leading organizational change. San Francisco: Jossey-Bass, 2003. 
HAIR, J. F. et al. Fundamentos de pesquisa em administração. São Paulo: Artmed, 2003. HOBBS, B.; AUBRY, M. A multi-phase research program investigating project management offices (pmos): the results of phase I. Project Management Journal, v. 38, n. I, p. 74-86, Mar. 2007.

IT GOVERNANCE INSTITUTE. Cobit 4.0: control objectives, management guidelines, maturity models. Rolling Meadows: IT Governance Institute, 2005.

JIANG, J. J.; KLEIN, G.; PICK, R. A. The impact of IS department organizational environments upon project team performances. Information \& Management, v. 40, n. 3, p. 2I3-220, Jan. 2003.

JUGDEV, K. Project management as a strategic asset: what does it look like and how do organizations get there? In: SLEVIN, D. P.; CLELAND, D. L.; PINTO, J. K. (Ed.). Innovations: project management research 2004. Newton Square: Project Management Institute, 2004. p. I6I-I74.

JUGDEV, K.; MÜLLER, R. A retrospective look at our evolving understanding of project success. Project Management Journal, v. 36, n. 4, p. I9-3I, Dec. 2005.

KERZNER, H. Gestão de projetos: as melhores práticas. Porto Alegre: Bookman, 2002.

KING, G.; KEOHANE, R. O. Designing social inquiry: scientific inference in qualitative research. Princeton: Princeton University Press, I994.

KNOPF, J. W. Doing a literature review. PS: Political Science \& Politics, v. 39, n. I, p. I27-I32, Jan. 2006. Disponívelem <http://journals.cambridge.org/action/displayIssue?jid=PSC\&volumeId=39\&issueId $=$ OI $>$. Acesso em: $I^{\circ}$ jul. 2007.

LIU, L.; YETTON, P. The contingent effects on project performance of conducting project reviews and deploying project management offices. IEEE Transactions on Engineering Management, v. 54, n. 4, p. 789-799, Nov. 2007.

MAXIMIANO, A. C. A.; ANSELMO, J. L. Escritório de gerenciamento de projetos: um estudo de caso. Revista de Administração da USP, v. 4I, n. 4, p. 394-403, out./nov./dez. 2006.

MEIRELLES, F. S. Gastos, investimentos e indicadores nas empresas: evolução de tendências. In: ALBERTIN, A. L.; MOURA, R. M. Tecnologia da informação. São Paulo: Atlas, 2004. p. I22.

MELLONE, N. P.; WHARTON, T. J. Strategies for MIS project selection. Journal of Systems Management, v. 35, n. 2, p. 28-33, Feb. 1984.

MILOSEVIC, D.; PATANAKUL, P. Standardized project management may increase development projects success. International Journal of Project Management, v. 23, n. 3, p. I8I-I92, Apr. 2005.

MUNNS, A. K.; BJEIRNI, B. F. The role of project management in achieving project success. International Journal of Project Management, v. I4, n. 2, p. 8I-87, Apr. I996.

PROJECT MANAGEMENT INSTITUTE. A guide to the project management body of knowledge. 3. ed. Newton Square, PA: Project Management Institute, 2004.

RAD, P. Is your organization a candidate for project management office (PMO)? AACE International Transactions, v. I, p. I-4, 200 I.

SAMBAMURTHY, V.; ZMUD, R. W. Arrangements for information technology governance: a theory of multiple contingencies. MIS Quarterly, v. 23, n. 2, p. 26I-290. June I999.

SAUER, C.; REICH, B. H. What do we want from a theory of project management? A response to Rodney Turner (Guest Editorial). International Journal of Project Management, v. 25, n. I, p. I-2, Jan. 2007.

SHENHAR, A. J. One size does not fit all projects: exploring classical contingency domains. Management Science, v. 47, n. 3, p. 394-4I4, Mar. 200I. 
SHENHAR, A. J; DVIR, D. Project management evolution: past history and future research directions. In: SLEVIN, D. P.; CLELAND, D. L.; PINTO, J. K. (Ed.). Innovations: project management research 2004. Newton Square: PMI, 2004. Chapter 4, p. 57-64.

SRIVANNABOON, S. The process of translating business strategy into project management actions. In: SLEVIN, D. P.; CLELAND, D. L.; PINTO, J. K. (Ed.). Innovations: Project Management Research 2004. Newton Square: PMI, 2004. p. I75-I92.

SUTTON, R. I.; STAW, B. M. What theory is not? Administrative Science Quarterly, v. 40, n. 3, p. 37I-384, Sept. I995.

TROCHIM, W. M. K.; DONNELLY, J. P. The research methods knowledge base. 3. ed. Mason, OH: Thomson, 2007.

WEBSTER, J.; WATSON, R. T. Analyzing the past to prepare for the future: writing a literature review. MIS Quarterly, v. 26, n. 2, p. xiii-xxiii, June 2002.

WEILL, P.; ROSS, J. W. IT governance: how top performers manage it decisions rights for superior results. Boston: Harvard Business School Press, 2004.

WHETTEN, D. A. What constitutes a theoretical contribution? Academy of Management Review, v. I4, n. 4, p. 490-495, Oct. I989. 